\title{
Validação cruzada de uma escala de clima organizacional
}

\author{
Jacob A. Laros \\ Katia E. Puente-Palacios \\ Universidade de Brasília
}

\begin{abstract}
Resumo
A estabilidade da estrutura fatorial de uma escala de clima organizacional com 66 itens foi investigada. A amostra de 61.349 respondentes foi dividida aleatoriamente em duas partes, a primeira para identificação da estrutura fatorial, a segunda para verificação da sua replicabilidade. O critério adotado para identificar o número de fatores resultou na extração de sete fatores. Para obter uma estrutura fatorial satisfatória 23 itens foram eliminados. Uma segunda análise fatorial dos 43 itens restantes indicou sete fatores explicando 63,4\% da variância. Uma análise fatorial de segunda ordem revelou um fator geral explicando 55,5\% da variância. Para verificar a estabilidade da estrutura fatorial, os mesmos procedimentos e critérios foram empregados na segunda amostra. Os resultados indicam uma grande estabilidade da estrutura fatorial hierárquica da escala de clima organizacional, com sete fatores de primeira ordem e um fator geral de segunda ordem.
\end{abstract}

Palavras-chave: validação cruzada; análise fatorial; validade de construto; estrutura fatorial hierárquica; clima organizacional

\begin{abstract}
Cross validation of a scale of organizational climate. The stability of the factor structure of a scale of organizational climate of 66 items was investigated. A sample of 61,349 respondents was randomly divided in two parts, the first to identify the factor structure, the second to verify its replicability. The criterion used to identify the number of factors resulted in the retention of seven factors. To obtain a satisfactory factor structure 23 items were excluded. A second factor analysis of the remaining 43 items indicated seven factors explaining $63.4 \%$ of the variance. Second-order factor analysis revealed one general factor accounting for $55.5 \%$ of the variance. To investigate the stability of the factor structure the same procedures and criteria were used with the second sample. The results indicate a high stability of the hierarchical factor structure of the scale of organizational climate consisting of seven first-order factors and one general second-order factor.
\end{abstract}

Key-words: cross validation; factor analysis; construct validity; hierarchical factor structure; organizational climate.

$\mathrm{U}$ ma pergunta que surge freqüentemente em relação aos instrumentos psicológicos é se a sua estrutura fatorial é replicável quando aplicados em outras amostras. Uma das técnicas mais utilizadas para verificar a estabilidade dessa estrutura é a validação cruzada. Esta técnica permite examinar se a estrutura identificada se repete quando investigada em uma segunda amostra. A sua utilização é importante tanto para soluções fatoriais exploratórias como para soluções fatoriais confirmatórias. Idealmente, o tamanho das amostras deveria ser suficientemente grande para permitir separar os respondentes aleatoriamente em dois grupos. Num deles seria realizada a derivação da estrutura fatorial e no outro a validação cruzada da solução fatorial identificada. Neste processo a designação aleatória é muito importante, uma vez que os grupos não podem diferir em relação às características sociodemográficas, pois isto pode afetar a estrutura fatorial (Floyd \& Widaman, 1995).
No campo da Psicologia Organizacional, o estudo do clima organizacional é uma área em franca expansão. O incremento do interesse pela adequada compreensão deste construto está acompanhado da necessidade de desenvolvimento de instrumentos de avaliação adequados que, sendo psicometricamente estáveis, permitam ao pesquisador coletar dados confiáveis sobre o fenômeno do seu interesse.

A importância deste campo pode ser verificada pela permanente publicação de revisões teóricas (Forehand \& Gillmer, 1964; James \& Jones, 1974; Moran \& Volkwein, 1992; Schneider \& Reichers, 1983), nas quais se procura estabelecer tanto as características do construto, quanto os seus limites conceituais. Esta evolução teórica que veio acompanhada de diversas pesquisas teóricas (Verbeke, Volgering, \& Hessels, 1998), bem como empíricas (James \& James, 1989; Koys \& DeCotiis, 1991), sugere a necessidade de desenvolvimento de escalas de mensuração estáveis. 
O objetivo perseguido com a realização deste estudo diz respeito à técnica de investigação da estabilidade psicométrica da escala analisada. Logo, características e limites teóricos do construto clima organizacional não serão discutidos, embora dados relativos à avaliação do clima organizacional tenham sido utilizados. Ainda com estas considerações, uma adequada definição do construto se faz necessária para facilitar a interpretação, por parte do leitor, dos dados aqui apresentados.

O clima organizacional é definido por Koys e DeCotiis (1991) como "fenômeno perceptual duradouro, construído com base na experiência, multi-dimensional e compartilhado pelos membros de uma unidade da organização, cuja função principal é orientar e regular os comportamentos individuais de acordo com os padrões determinados por ela” (p. 266). A importância da sua investigação é decorrente da influência que exerce tanto sobre o comportamento como sobre o desempenho dos trabalhadores, podendo refletir-se na efetividade da organização. Pesquisas deste campo investigam as relações entre clima organizacional e envolvimento (Shadur, Kienzle, \& Rodwell, 1999; Toulson \& Smith, 1994), práticas administrativas (Lin, 1999; Toulson \& Smith, 1994), estresse ocupacional (Hemingway \& Smith, 1999) e comportamentos organizacionais e individuais (Brown \& Leigh, 1996; Welsh \& LaVan, 1981). Assim, é possível verificar o amplo leque de abrangência da influência exercida pelo clima no cenário organizacional. Decorrente desta constatação pode ser visualizada a importância do desenvolvimento de instrumentos de mensuração adequados que, possuindo uma estrutura estável, permitam a obtenção de resultados conclusivos e generalizáveis neste campo de conhecimento.

\section{Método}

Para a realização da presente pesquisa foram utilizados dados secundários, provenientes do diagnóstico do clima organizacional realizado por uma instituição financeira, em nível nacional. Os dados fornecidos pela instituição foram exclusivamente as respostas dadas aos itens da escala de clima, sem qualquer informação relacionada às características sociodemográficas ou funcionais dos respondentes. A amostra inicial consistiu de 63.390 respondentes. Considerando o tamanho da amostra, optou-se pela exclusão dos questionários que apresentassem uma ou mais perguntas sem resposta. Assim, foram excluídos 2.041 respondentes, que correspondem a 3,2\% do total. As análises estatísticas posteriores foram realizadas em duas amostras extraídas aleatoriamente da amostra total composta por 61.349 respondentes.

\section{Instrumento}

Os dados foram levantados utilizando uma versão modificada do questionário de avaliação do clima organizacional, desenvolvido pela Empresa Brasileira de Pesquisa Agropecuária - EMBRAPA - (Sá Leitão, Guimarães, \& Azevedo Rosal, 1998). Essa versão do instrumento está composta por 66 itens $^{1}$ distribuídos em 8 fatores: Desenvolvi- mento Profissional $(\mathrm{a}=0,91)$, Clareza Organizacional e Padrão de Desempenho $(\mathrm{a}=0,70)$, Recompensas e Volume de Trabalho $(\mathrm{a}=0,72)$, Condições de Trabalho $(\mathrm{a}=0,78)$, Estilo de Gerência $(\mathrm{a}=0,70)$, Reconhecimento $(\mathrm{a}=0,89)$, Comprometimento Organizacional $(\mathrm{a}=0,87)$ e Autogestão de Equipe $(\mathrm{a}=$ 0,83). Os 8 fatores em conjunto explicam, segundo relatório interno da empresa, 54\% da variância total.

\section{Análise de dados e resultados}

Considerando que o objetivo da validação cruzada da estrutura fatorial de um instrumento é a verificação da estabilidade da solução fatorial em grupos diferentes, o banco de dados foi dividido aleatoriamente em duas partes, sendo que cada uma esteve composta por, aproximadamente, 50\% do total de respondentes (30.684 e 30.665). As duas amostras foram analisadas de maneira independente seguindo os mesmos passos e procedimentos. Contudo, dado o objetivo perseguido com a realização da presente pesquisa, os resultados encontrados nas análises realizadas nas duas amostras serão apresentados de maneira simultânea.

O primeiro passo nas análises da estrutura do instrumento foi a verificação da fatorabilidade da matriz de correlações de cada amostra, pois se as variáveis não têm variância em comum, a análise fatorial não faz sentido. Para verificar a fatorabilidade da matriz de correlações das duas amostras, vários procedimentos foram executados: (1) inspeção da matriz de correlações; (2) verificação do determinante da matriz; (3) cálculo do índice de adequação da amostra de KaiserMeyer-Olkin (KMO).

Para a inspeção da matriz de correlações foram seguidas as recomendações de Clark e Watson (1995), considerando o vasto tamanho da matriz de intercorrelações (2.154 correlações inter-item). Desta forma, optou-se pelo cálculo da média aritmética das correlações inter-item. Os resultados encontrados nas duas amostras foram iguais. A média aritmética das correlações entre itens foi 0,32, com valores entre 0,03 e 0,76 enquanto que o valor da variância foi 0,015. Estes dados evidenciam que os itens são suficientemente relacionados entre si, justificando a fatorização da matriz.

O cálculo do determinante da matriz revelou valores baixos, mas diferentes de zero nas duas amostras (2,314E-17 na primeira e 1,694E-17 na segunda). Disto pode ser concluído que fatorar a matriz é pertinente. O cálculo do índice de adequação da amostra, o KMO, revelou uma fatorabilidade do instrumento muito satisfatória (=0,98 nas duas amostras). $\mathrm{O}$ índice KMO está baseado nas correlações parciais entre as variáveis, quanto mais próximo de 1 , maior a comunalidade entre as variáveis e melhor a fatorabilidade do conjunto.

Depois da verificação da fatorabilidade da matriz, a consistência interna do conjunto de itens foi investigada. A importância desta investigação, segundo Laros (no prelo), está sustentada no fato de que, se um item não mostra pelo menos uma correlação moderada (por exemplo $r=0,20$ ) com os outros itens do construto, provavelmente terá um desempenho pobre na análise fatorial. Nas duas amostras com as quais a presente pesquisa foi realizada, o valor da média arit- 
mética da correlação item-total corrigida foi de 0,56, com um desvio padrão de 0,11 , enquanto que o coeficiente de fidedignidade (a) foi de 0,97.

A seguir foi definido o número de fatores a extrair. Na análise fatorial, esta decisão é de vital importância, uma vez que a sub ou super-extração distorcerá qualquer resultado de análise posterior (Fava \& Velicer, 1996). Pesquisa com dados simulados (Zwick \& Velicer, 1986) mostra que o critério do eigenvalue maior do que 1,0, de Guttman-Kaiser (Guttman, 1954; Kaiser, 1960) é adequado em 22\% dos casos; o critério baseado no teste qui-quadrado, de Barlett (1950), é adequado em 30\% dos casos; o critério baseado no teste scree, de Cattell (1966), é adequado em 57\% dos casos; o critério de Velicer (1976), baseado nas correlações parciais, é adequado em 84\% dos casos e o critério baseado na AP, de Horn (1965), é adequado em $92 \%$ dos casos. Considerando estes resultados, o critério da análise paralela (AP) foi o escolhido para definir o número de fatores a extrair.

$\mathrm{Na}$ AP os eigenvalues de uma matriz de correlação de dados aleatórios são comparados com os da matriz empírica calculada pela análise dos componentes principais. Este procedimento de comparação dos eigenvalues, focaliza a magnitude de variância explicada pelos componentes. Segundo Reise, Waller e Comrey (2000), é pertinente reter um fator apenas na medida em que este explica maior variância do que o fator correspondente nos dados aleatórios. Assim, no momento em que o valor do eigenvalue dos dados aleatórios é superior ao dos dados empíricos, não é mais adequado reter esse fator.

Para identificar o valor dos eigenvalues aleatórios, o software RanEigen (Enzmann, 1997) foi utilizado. A Tabela 1 mostra os eigenvalues empíricos das duas amostras assim como os aleatórios. Conforme o leitor pode verificar, o componente 7 é o último em que os eigenvalues empíricos são superiores aos aleatórios. Já no componente 8, os valores empíricos são menores do que o valor aleatório. Estes dados mostram claramente a existência de sete fatores.

Uma vez definido o número de fatores a extrair, utilizou-se o método de análise fatorial dos eixos principais (análise fatorial comum) na extração dos fatores. Os fatores extraídos foram submetidos à rotação para favorecer a sua interpretabilidade. O método de rotação definido para esta análise foi oblíquo (oblimin). Reise et al. (2000) defendem a escolha da rotação oblíqua com base nos seguintes argumentos: (1) produz soluções ortogonais quando apropriadas; (2) permite a fatorização dos fatores de primeira ordem para a identificação de um possível fator de segunda ordem. Com a rotação ortogonal esta possibilidade é inviabilizada. De maneira adicional, pode ser considerado o argumento teórico de que os fatores constitutivos do clima organizacional são relacionados entre si (James \& Jones, 1974).

Após ter sido realizada a rotação, procurou-se obter uma solução fatorial satisfatória. Segundo Thurstone (1947) a interpretabilidade da estrutura fatorial é otimizada quando a estrutura é simples. Em relação à análise fatorial, uma estrutura simples significa que os itens apresentam somente uma carga fatorial principal, enquanto as outras cargas são próximas de zero. A inspeção das cargas fatoriais dos 66 itens revelou que nem todos eles estavam contribuindo positivamente na mensuração dos fatores. Estes itens foram excluídos utilizando os seguintes critérios: (1) valor absoluto da carga fatorial principal do item menor do que 0,32. Tabachnick e Fidell, (1996) propõem este valor como o mínimo aceitável para que um item seja considerado representante legítimo do construto mensurado pelo fator; (2) diferença entre os valores absolutos das cargas fatoriais principais de um item menor do que 0,10 . Gorsuch (1983) aponta que os itens não podem ter cargas principais similares em dois ou mais fatores e (3) ausência de similaridade entre o conteúdo do item e o domínio teórico do construto. Smith e McCarthy (1995) enfatizam que tão importante quanto investigar as características psicométricas do item, é verificar se consegue representar, de maneira acurada, o conteúdo abordado pelo fator.

Este conjunto de critérios foi aplicado de maneira iterativa até que nenhum item apresentasse os critérios de exclusão. No total, 23 dos 66 itens foram eliminados, restando 43 itens na solução fatorial definitiva. A aplicação destes critérios resultou na eliminação dos mesmos itens nas duas amostras. Com os 43 itens restantes, uma nova análise fatorial foi realizada. Os eigenvalues encontrados nas duas amostras, a porcentagem de variância explicada pelos fatores antes da rotação e a fidedignidade de cada fator podem ser vistas na Tabela 2.

Tabela 1

Eigenvalues empíricos e aleatórios dos primeiros dez componentes nas duas amostras

\begin{tabular}{|c|c|c|c|c|c|c|c|c|c|c|}
\hline \multirow{2}{*}{ Eigenvalue } & \multicolumn{10}{|c|}{ Componentes } \\
\hline & 1 & 2 & 3 & 4 & 5 & 6 & 7 & 8 & 9 & 10 \\
\hline Empírico & $\begin{array}{c}22,76 \\
(22,98)\end{array}$ & $\begin{array}{l}4,49 \\
(4,51)\end{array}$ & $\begin{array}{c}2,70 \\
(2,69)\end{array}$ & $\begin{array}{l}2,50 \\
(2,52)\end{array}$ & $\begin{array}{c}1,68 \\
(1,68)\end{array}$ & $\begin{array}{c}1,54 \\
(1,55)\end{array}$ & $\begin{array}{c}1,35 \\
(1,31)\end{array}$ & $\begin{array}{c}1,22 \\
(1,18)\end{array}$ & $\begin{array}{c}1,08 \\
(1,12)\end{array}$ & $\begin{array}{c}1,00 \\
(1,05)\end{array}$ \\
\hline Aleatório & 1,37 & 1,34 & 1,32 & 1,30 & 1,29 & 1,27 & 1,26 & 1,24 & 1,23 & 1,22 \\
\hline
\end{tabular}

Nota. Os eigenvalues empíricos relatados são as médias dos valores de 4 sub-amostras de 2.000 casos da amostra 1 (30.684 casos) e 2 (30.665 casos); os valores entre parênteses correspondem à amostra 2 . Os eigenvalues aleatórios foram calculados com base numa amostra de 2.000 casos. 
Tabela 2

Eigenvalues, variância explicada pelos fatores antes da rotação, coeficiente de fidedignidade e correlação média entre os itens dos sete fatores. Os dados entre parênteses correspondem à segunda amostra

\begin{tabular}{lcccccccc}
\hline & Fator 1 & Fator 2 & Fator 3 & Fator 4 & Fator 5 & Fator 6 & Fator 7 & Total \\
\hline Eigenvalue & 15,67 & 3,47 & 2,27 & 2,10 & 1,48 & 1,23 & 1,07 & 27,29 \\
& $(15,77)$ & $(3,51)$ & $(2,22)$ & $(2,07)$ & $(1,47)$ & $(1,22)$ & $(1,09)$ & $(27,35)$ \\
\% variância & 36,44 & 8,06 & 5,20 & 4,89 & 3,44 & 2,86 & 2,47 & 63,38 \\
explicada & $(36,66)$ & $(8,16)$ & $(5,16)$ & $(4,81)$ & $(3,43)$ & $(2,84)$ & $(2,53)$ & $(63,59)$ \\
& 0,95 & 0,88 & 0,88 & 0,86 & 0,73 & 0,87 & 0,82 & 0,95 \\
Alfa $(\alpha)$ & $(0,95)$ & $(0,88)$ & $(0,88)$ & $(0,87)$ & $(0,73)$ & $(0,87)$ & $(0,82)$ & $(0,96)$ \\
Correlação & 0,60 & 0,53 & 0,64 & 0,45 & 0,41 & 0,69 & 0,48 & 0,34 \\
entre itens & $(0,60)$ & $(0,53)$ & $(0,64)$ & $(0,46)$ & $(0,41)$ & $(0,69)$ & $(0,49)$ & $(0,34)$ \\
\hline \multirow{2}{*}{$\mathrm{N}$ itens } & 12 & 7 & 4 & 8 & 4 & 3 & 5 & 43 \\
\hline
\end{tabular}

A inspeção da Tabela 2 mostra que os eigenvalues dos sete fatores nas duas amostras são praticamente iguais. Este resultado pode ser considerado um primeiro indicador da semelhança da estrutura fatorial nas amostras em que foi investigada. O primeiro fator explica, antes da rotação, aproximadamente 4,5 vezes mais variância do que o segundo. Este dado sinaliza a presença de um fator de segunda ordem. De maneira adicional, pode ser observado que em ambas as amostras os 7 fatores explicam em conjunto aproximadamente $64 \%$ da variância. Os valores do alfa de Cronbach dos fatores oscilam entre 0,73 e 0,95. A este respeito, Clark e Watson (1995) afirmam que a correlação média entre itens é um indicador mais adequado da consistência interna do que o coeficiente alfa, que é afetado pelo número de itens. Cortina (1993) ressalta adicionalmente, que o coeficiente alfa perde a sua utilidade em escalas compostas por mais de 40 itens. A Tabela 2 mostra valores médios das correlações entre itens nos sete fatores variando de 0,41 a 0,69. Estes valores revelam uma consistência interna satisfatória de acordo com os critérios de Clark e Watson (1995), que manifestam que a correlação média entre itens deve ficar entre 0,15 e 0,50.

A distribuição dos 43 itens nos sete fatores está disposta na Tabela 3. A inspeção dessa tabela permite verificar que praticamente todas as cargas (40 de 43 itens) estão acima de 0,40 . Isto pode ser considerado um indicador da qualidade da solução fatorial identificada. De maneira adicional, pode ser observado que as cargas fatoriais dos itens, nas duas amostras, são muito semelhantes.

Observando-se a Tabela 3, pode ser verificado que a média aritmética das cargas dos itens que compõem os fatores apresenta valores absolutos entre 0,42 e 0,80 ( 0,43 e 0,80 na segunda amostra). $\mathrm{O}$ valor mais baixo refere-se ao fator $7 \mathrm{e}$ o mais elevado ao fator 3. Estes resultados permitem concluir que os itens que compõem o fator 3 são indicadores mais fortes do construto subjacente, enquanto os itens do fator 7 são representantes mais fracos do construto avaliado. $\mathrm{Na}$ tabela também pode ser observado que os itens que compõem o fator 1 são mais heterogêneos do que os dos outros fatores. A carga fatorial mínima é 0,44 e a máxima e 0,87 (0,45 e 0,83 na segunda amostra). Estes dados evidenciam a heterogeneidade das cargas do fator, que pode ser esperada considerando a grande quantidade de itens que o compõem.

Além de verificar as características psicométricas para justificar a associação dos itens nos fatores, foi investigado se os itens representam o construto teórico de maneira adequada. Todos os conjuntos de itens que compõem os sete fatores mostraram um conteúdo teórico pertinente ao construto abordado. Os resultados da interpretação teórica dos fatores podem ser vistos na Tabela 4.

Após a interpretação teórica dos fatores, a correlação entre eles foi verificada. A média aritmética das correlações entre os sete fatores foi de 0,37 na primeira amostra e 0,38 na segunda. Isto pode ser considerado um indicador da presença de um ou mais fatores de segunda ordem. Para definir o número adequado de fatores a reter, novamente o critério de AP foi empregado. Este procedimento resultou na identificação de um fator geral de segunda ordem. Uma análise fatorial dos eixos principais foi realizada considerando os sete fatores de primeira ordem como variáveis. As cargas fatoriais no fator de segunda ordem podem ser observadas na Tabela 4.

A fidedignidade do fator de segunda ordem, calculada através do coeficiente alfa estratificado (Nunnally, 1978) foi 0,95 na primeira amostra e 0,96 na segunda. A fidedignidade do fator único, considerando os sete fatores de primeira ordem como itens, foi 0,82 nas duas amostras. Este índice de fidedignidade, também chamado de "generalizabilidade" (generalizability), sempre mostra valores mais baixos que a fidedignidade tradicional (Laros \& Tellegen, 1991) e pode ser interpretado como a correlação esperada entre instrumentos medindo o mesmo construto geral. $\mathrm{O}$ valor 0,82 encontrado no estudo atual pode, portanto, ser interpretado como a correlação esperada entre a escala de clima organizacional revisada, composta por 43 itens, e qualquer outro instrumento de clima organizacional.

Conforme mostra a Tabela 4, os fatores 1, 4, 6 e 7 podem ser considerados os melhores representantes do 
Tabela 3

Cargas fatoriais dos itens da escala de clima organizacional depois da extração com análise fatorial dos eixos principais e rotação oblíqua nas duas amostras. As cargas fatoriais dos itens na segunda amostra estão dispostas entre parênteses

\begin{tabular}{|c|c|c|c|c|c|c|c|}
\hline Item & Fator 1 & Fator 2 & Fator 3 & Fator 4 & Fator 5 & Fator 6 & Fator 7 \\
\hline 06 & $0,68(0,67)$ & & & & & & \\
\hline 10 & $0,55(0,57)$ & & & & & & \\
\hline 15 & $0,44(0,45)$ & & & & & & \\
\hline 23 & $0,87(0,83)$ & & & & & & \\
\hline 25 & $0,70(0,68)$ & & & & & & \\
\hline 27 & $0,64(0,63)$ & & & & & & \\
\hline 30 & $0,79(0,78)$ & & & & & & \\
\hline 37 & $0,57(0,55)$ & & & & & & \\
\hline 40 & $0,83(0,85)$ & & & & & & \\
\hline 43 & $0,76(0,76)$ & & & & & & \\
\hline 45 & $0,80(0,81)$ & & & & & & \\
\hline 49 & $0,81(0,83)$ & & & & & & \\
\hline 28 & & $0,79(0,78)$ & & & & & \\
\hline 33 & & $0,55(0,54)$ & & & & & \\
\hline 44 & & $0,73(0,72)$ & & & & & \\
\hline 46 & & $0,75(0,75)$ & & & & & \\
\hline 55 & & $0,76(0,76)$ & & & & & \\
\hline 58 & & $0,57(0,58)$ & & & & & \\
\hline 64 & & $0,51(0,50)$ & & & & & \\
\hline 24 & & & $0,76(0,76)$ & & & & \\
\hline 54 & & & $0,74(0,74)$ & & & & \\
\hline 63 & & & $0,87(0,87)$ & & & & \\
\hline 65 & & & $0,81(0,81)$ & & & & \\
\hline 09 & & & & $0,59(0,61)$ & & & \\
\hline 16 & & & & $0,67(0,68)$ & & & \\
\hline 20 & & & & $0,77(0,77)$ & & & \\
\hline 38 & & & & $0,38(0,37)$ & & & \\
\hline 39 & & & & $0,36(0,37)$ & & & \\
\hline 53 & & & & $0,43(0,42)$ & & & \\
\hline 59 & & & & $0,76(0,77)$ & & & \\
\hline 60 & & & & $0,69(0,70)$ & & & \\
\hline 03 & & & & & $0,44(0,44)$ & & \\
\hline 12 & & & & & $0,45(0,45)$ & & \\
\hline 19 & & & & & $0,80(0,81)$ & & \\
\hline 35 & & & & & $0,75(0,74)$ & & \\
\hline 07 & & & & & & $-0,65(-0,64)$ & \\
\hline 31 & & & & & & $-0,72(-0,72)$ & \\
\hline 36 & & & & & & $-0,78(-0,78)$ & \\
\hline 02 & & & & & & & $-0,48(-0,49)$ \\
\hline 05 & & & & & & & $-0,45(-0,45)$ \\
\hline 08 & & & & & & & $-0,40(-, 041)$ \\
\hline 11 & & & & & & & $-0,41(-, 043)$ \\
\hline 51 & & & & & & & $-0,38(-, 038)$ \\
\hline Carga & 0,70 & 0,67 & 0,80 & 0,58 & 0,61 & $-0,72$ & $-0,42$ \\
\hline Média & $(0,70)$ & $(0,66)$ & $(0,80)$ & $(0,57)$ & $(0,61)$ & $(-0,71)$ & $(-0,43)$ \\
\hline
\end{tabular}

Tabela 4

Conteúdo avaliado pelos fatores de primeira ordem e suas cargas fatoriais no fator de segunda ordem

\begin{tabular}{clc}
\hline Fator & \multicolumn{1}{c}{ Conteúdo } & Cargas \\
\hline 1 & Gerenciamento das relações interpessoais & $0,79(0,78)$ \\
2 & Comprometimento organizacional & $0,66(0,66)$ \\
3 & Carga de trabalho & $0,48(0,49)$ \\
4 & Trabalho em equipe & $0,74(0,74)$ \\
5 & Suporte material & $0,58(0,59)$ \\
7 & Valorização do trabalho & $0,75(0,75)$ \\
\hline
\end{tabular}


construto subjacente ao fator geral. Apresentam cargas mais elevadas, oscilando entre 0,74 e 0,82 . Já os fatores 2 , 3 e 5 são os piores representantes, pois as suas cargas estão entre 0,48 e 0,66. Aparentemente estes últimos estão medindo aspectos menos centrais do construto clima organizacional. Em relação ao conteúdo abordado pelos fatores com cargas menores (comprometimento organizacional, carga de trabalho e suporte organizacional), Puente-Palacios (2002) questiona a pertinência teórica da sua inclusão como elementos constitutivos do clima organizacional.

\section{Discussão e conclusões}

A realização deste estudo permitiu verificar que a escala de clima organizacional exibe uma estrutura fatorial hierárquica com sete fatores de primeira ordem e um de segunda ordem. As análises realizadas com amostras grandes (mais de 30.000 casos) mostraram claramente a presença de 23 itens que podem ser considerados problemáticos. Em decorrência desta constatação, esses itens foram excluídos das análises posteriores. Portanto, a escala de clima organizacional assim revisada está composta por 43 itens.

Os sete fatores de primeira ordem explicaram aproximadamente $63 \%$ da variância, quase $10 \%$ a mais do que os oito fatores identificados na escala modificada e quase $18 \%$ a mais do que a escala original de Sá Leitão et al. (1998). Tomando como base a estrutura fatorial simples identificada, assim como o incremento na porcentagem da variância explicada pela escala revisada, é considerado pertinente afirmar que a validade de construto desta escala é melhor do que a da original, bem como da modificada, utilizada pela instituição financeira. O construto mensurado, clima organizacional, é mais adequadamente abordado na versão da escala composta por 43 itens.

Para a realização desta pesquisa, o uso da análise paralela (AP) foi um procedimento essencial na identificação da estrutura fatorial, no que diz respeito à definição do número de fatores que deveriam ser extraídos. Esta identificação é de grande importância porque a extração de mais ou menos fatores do que o adequado, distorcerá qualquer resultado de análise posterior e inviabilizará a replicação da estrutura fatorial, portanto, a realização de comparações entre grupos.

De maneira adicional, a realização da presente pesquisa permitiu identificar a estrutura hierárquica da escala. Ficou evidenciado que a escala de clima organizacional está medindo um fator geral composto por sete sub-fatores específicos. A análise da estrutura hierárquica revelou, também, que quatro dos sete fatores encontrados podem ser considerados melhores representantes do construto geral de clima organizacional, enquanto os três fatores restantes contribuem menos na mensuração do fator geral. A partir desta análise é possível verificar que a investigação da presença de fatores de segunda ordem contribui à compreensão da estrutura fatorial do instrumento e favorece o desenvolvimento teórico do campo investigado, fornecendo subsídios adicionais sobre os elementos constitutivos centrais e periféricos do construto em questão.
A realização do presente estudo não apenas permitiu a identificação da estrutura fatorial da escala de clima organizacional. Revelou também a estabilidade da escala a partir da utilização de uma amostra de derivação e uma de validação cruzada. A aplicação dos mesmos procedimentos nas duas amostras mostrou a estabilidade da estrutura fatorial encontrada.

Uma vez que não tivemos acesso aos dados sociodemográficos dos respondentes não foi possível investigar a generalidade da estrutura fatorial encontrada. Pesquisas futuras precisam mostrar se essa estrutura fatorial também pode ser generalizada para amostras compostas por indivíduos com características diferentes em termos de sexo, idade, escolaridade ou outras. Somente após essa verificação, a generalidade da estrutura fatorial da escala de avaliação do clima organizacional poderá ser defendida.

Finalmente, um resultado adicional atingido com a realização da presente pesquisa é a constatação da adequação dos procedimentos adotados. Na realização de pesquisas que visam identificar a estrutura fatorial de uma escala, o pesquisador se depara com a necessidade de tomar diversas decisões. A este respeito, Laros (no prelo) manifesta que as decisões a serem implementadas são de suma importância, pois virão afetar profundamente a estrutura encontrada. Caso se tomem decisões erradas, a estabilidade da solução identificada será comprometida.

\section{Referências}

Barlett, M. S. (1950). Tests of significance in factor analysis. British Journal of Psychology, 3, 77-85.

Brown, S., \& Leigh, T. (1996). A new look at psychological climate and its relationship to job involvement, effort, and performance. Journal of Applied Psychology, 81, 358-368.

Cattell, R. B. (1966). The scree test for the number of factors. Multivariate Behavioral Research, 1, 245-276.

Clark, A. C., \& Watson, D. (1995). Constructing validity: basic issues in objective scale development. Psychological Assessment, 7, 309-319.

Cortina, J. M. (1993). What is coefficient alpha? An examination of theory and applications. Journal of Applied Psychology, 78, 98-104.

Enzmann, D. (1997). RanEigen: a program to determine the parallel analysis criterion for the number of principal components. Applied Psychological Measurement, 21, 232.

Fava, J. L., \& Velicer W. F. (1996). The effect of underextraction in factor and component analysis. Educational and Psychological Measurement, 56, 907 929.

Floyd, F. J., \& Widaman K. F. (1995). Factor analysis in the development and refinement of clinical assessment instruments. Psychological Assessment, 7, 286-299.

Forehand, G., \& Gillmer, H. (1964). Environmental variation in studies of organizational behavior. Psychological Bulletin, 62, 361-383.

Gorsuch, R. L. (1983). Factor analysis ( $2^{a}$ ed.). Hillsdale, New Jersey: Lawrence Erlbaum.

Guttman, L. (1954). Some necessary conditions for common factor analysis. Psychometrika, 19, 149-162.

Hemingway, M., \& Smith, C. (1999). Organizational climate and organizational stressors as predictors of withdrawal behaviors and injuries in nurses. Journal of Occupational and Organizational Psychology, 72, 285-299. 
Horn, J. L. (1965). A rationale and test for the number of factors in factor analysis. Psychometrika, 30, 179-185.

James. L., \& James, L. (1989). Integrating work environment perceptions: explorations into the measurement of meaning. Journal of Applied Psychology, 74, 739-751.

James, L., \& Jones, A. (1974). Organizational climate: a review of theory and research. Psychological Bulletin, 81, 1096-1112.

Kaiser, H. F. (1960). The application of electronic computers to factor analysis. Educational and Psychological Measurement, 20, 141-151.

Koys, D., \& DeCotiis, T. (1991). Inductive measures of psychological climate. Human Relations, 44, 265-285.

Laros, J. A. (no prelo). O uso da análise fatorial: algumas diretrizes para pesquisadores. In L. Pasquali (Org.), Análise fatorial para pesquisadores. Brasília: Editora da Universidade de Brasília.

Laros, J. A., \& Tellegen, P. J. (1991). Construction and validation of the SON$R$ 51/2-17, the Snijders-Oomen non verbal intelligence test. Groningen: Wolters-Noordhoff.

Lin, C. (1999). The association between organizational climate and quality management practices: an empirical study on small- and medium-sized manufacturing companies in Taiwan. Total Quality Management, 10, 863-868.

Moran, E., \& Volkwein, G. (1992). The cultural approach to the formation of organizational climate. Human Relations, 45, 19-47.

Nunnally, J. C. (1978). Psychometric theory ( $2^{\mathrm{a}}$ ed.). Nova York: McGraw-Hill.

Puente-Palacios, K. E. (2002). Abordagens teóricas e dimensões empíricas do conceito de clima organizacional. Revista de Administração-RAUSP, 37, 96-104.

Reise, S. P., Waller, N. G., \& Comrey, A. L. (2000). Factor analysis and scale revision. Psychological Assessment, 12, 287-297.

Sá Leitão, J., Guimarães, T., \& de Azevedo Rosal, M. (1998). Metodologia de diagnóstico de clima organizacional em ambiente de inovação tecnológica
[Texto completo]. Associação Nacional dos Programas de Pós-Graduação em Administração (Org.), XXII Encontro Anual da Associação Nacional dos Programas de Pós-Graduação em Administração - ENANPAD (Texto em CD-Rom, p. 1-15). Foz de Iguaçú: Autor.

Shadur, M., Kienzle, R., \& Rodwell, J. (1999). The relationship between organizational climate and employee perceptions of involvement. Group and Organizational Management, 24, 479-503.

Schneider, B., \& Reichers, A. (1983). On etiology of climates. Personnel Psychology, 36, 19-39.

Smith, G. T., \& McCarthy, D. M. (1995). Methodological considerations in the refinement of clinical assessment instruments. Psychological Assessment, 7, 300-308.

Tabachnick, B. G., \& Fidell, L. S. (1996). Using multivariate statistics ( $3^{a}$ ed.). Nova York: HarperCollins.

Thurstone, L. L. (1947). Multiple factor analysis. Chicago: University of Chicago Press.

Toulson, P., \& Smith, M. (1994). The relationship between organizational climate and employee perceptions of personnel management practices. Public Personnel Management, 23, 453-468.

Velicer, W. F. (1976). Determining the number of components from the matrix of partial correlations. Psychometrika, 41, 321-327.

Verbeke, W., Volgering, M., \& Hessels, M. (1998). Exploring the conceptual expansion within the field of organizational behaviour: organizational climate and organizational culture. Journal of Management Studies, 35, 303-329.

Welsh, H., \& LaVan, H. (1981). Interrelationship between organizational commitment and job characteristics, job satisfaction, professional behavior, and organizational climate. Human Relations, 34, 1079-1089.

Zwick, R., \& Velicer, W. F. (1986). Comparison of five rules for determining the number of components to retain. Psychological Bulletin, 99, 432-442.

\section{Nota}

1 O instrumento relatado pelos autores em 1998, estava composto por 70 itens e sete fatores. Contudo, no processo de validação psicométrica realizado pela instituição financeira, algumas mudanças foram realizadas, resultando num instrumento composto por 66 itens e oito fatores.

Jacob A. Laros, doutor pela Rijksuniversiteit Groningen, RUG (Holanda), é professor no departamento de Psicologia Social e do Trabalho, Instituto de Psicologia, Universidade de Brasília. Endereço para correspondência: Universidade de Brasília, Instituto de Psicologia, Departamento de Psicologia Social e do Trabalho; Brasília, DF; CEP 70.910-900.E-mail: jalaros@unb.br

Katia E. Puente-Palacios, doutora em Psicologia Organizacional e do Trabalho pela Universidade de Brasília, é professora no Departamento de Psicologia Social e do Trabalho do Instituto de Psicologia da Universidade de Brasília. 